\title{
Focused Ion Beams in Biology: How the Helium Ion Microscope and FIB/SEMs Help Reveal Nature's Tiniest Structures
}

\author{
Annalena Wolff ${ }^{*}$, Nico Klingner ${ }^{2}$, William Thompson ${ }^{3}$ Yinghong Zhou ${ }^{4,6}$, Jinying Lin ${ }^{5,6}$, Yong Y \\ Peng $^{7}$, John A.M. Ramshaw ${ }^{7,8}$ and Yin $\mathrm{Xiao}^{4,6}$ \\ ${ }^{1 .}$ Central Analytical Research Facility, Institute for Future Environments, Queensland University of \\ Technology (QUT), Brisbane, Australia. \\ 2. Helmholtz-Zentrum Dresden-Rossendorf (HZDR), Dresden, Germany. \\ 3. HeelionicsLLC, Los Altos, CA, USA. \\ 4. Institute of Health and Biomedical Innovation, Queensland University of Technology (QUT), \\ Brisbane, Australia. \\ 5. Department of Implantology, Xiamen Stomatological Research Institute, Xiamen Stomatological \\ Hospital, Fujan, China. \\ 6. The Australia-China Centre for Tissue Engineering and Regenerative Medicine (ACCTERM), \\ Queensland University of Technology, Brisbane, Australia. \\ 7. CSIRO Manufacturing, Bayview Avenue, Clayton, Australia. \\ 8. Department of Surgery, St. Vincent's Hospital, \\ *Corresponding author: Annalena.wolff@qut.edu.au
}

Focused Ion Beam (FIB) devices such as the Helium Ion Microscope (HIM) as well as FIB/SEMs have sparked great interest within the biological sciences in recent years. The HIM allows high resolution imaging of uncoated non-conductive samples while the FIB/SEM (FIB/SEMs combine a focused ion beam with a Scanning Electron Microscope (SEM)) allows to prepare TEM lamellae, 3D reconstruct the sample or reveal sub surface structures with nanometre precision. FIB/SEMs have become the "go to" tool in the materials sciences and semiconductor industry. Despite these unique capabilities, it is not yet fully established in the biological sciences [1-3]. This is predominantly due to the heat-induced damage from the ion beam when processing soft materials including biological samples.

This presentation shows how the HIM as well as FIB/SEMs can be used in biological sciences to reveal nature's tiniest structures (Figure 1). The presented work then focuses on the underlying ion-solid interactions and the effect of processing parameters on heating induced by ion beams. The work presented here deals with gallium ion solid interactions, however the broader results are applicable to any type of FIB including the helium ion microscope (HIM) and plasma FIBs. The interactions of gallium ions in skin were simulated using Monte Carlo methods for different beam parameters. The program SRIM [4] was used to obtain theoretical results which permit estimation of the ion beam induced temperature increases, using the physical principles of Fourier's law of conductive heat transfer. The model suggests that the ion beam induced increase in temperature can be reduced by:

1. Reducing the local dose rate (smaller aperture/ion beam current)

2. Reducing the local dose (smaller aperture/ion beam current, introducing beam blur, reducing the beam overlap)

The technique was tested on collagen, a soft biological material which is commonly used in biomedical applications. Collagen was chosen as a suitable test sample as it loses its fibrillary structure when denaturated by heat, permitting damage to easily be recognized. Cross-sections and TEM lamellas were 
prepared from non-embedded collagen with conventional FIB processing parameters (see Figure 2 left) as well as heat reducing FIB parameters (see Figure 1 right).

The results also show that heat damage can be prevented by reducing the local dose rate and area underneath the ion beam. A TEM comparison of a microtome prepared lamella and a FIB prepared lamella (using different heat reducing parameters) shows that the fibrillar structures can be maintained, and heat damage avoided. The approach described here can be used to determine suitable parameters for other soft materials [5].

\section{References:}

[1] Drobne et al., Microscopy Research and Technique 70 (2007), p. 895.

[2] Stokes et al., Journal of Physics: Conference Series 26 (2006), p. 50.

[3] Bandara et al., ACS Applied Materials \& Interfaces 9 (2017), p. 6746.

[4] Ziegler et al., www.srim.org.

[5] The authors acknowledge scientific and technical assistance of Peter Hines, Jamie Riches, Rachel Hancock, and Ning Liu and the facilities at the Australian Microscopy \& Microanalysis Research Facility (AMMRF) at the Central Analytical Research Facility (CARF), Queensland University of Technology, Brisbane, Australia.

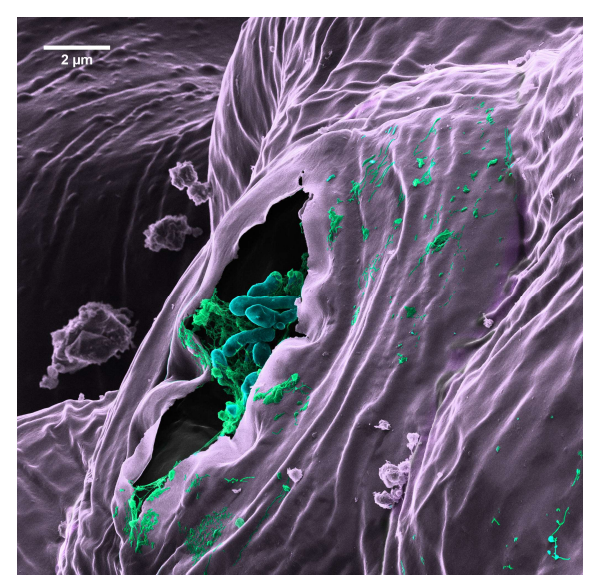

Figure 1. False colour HIM micrograph of agrobacterium entering a plant through stomata
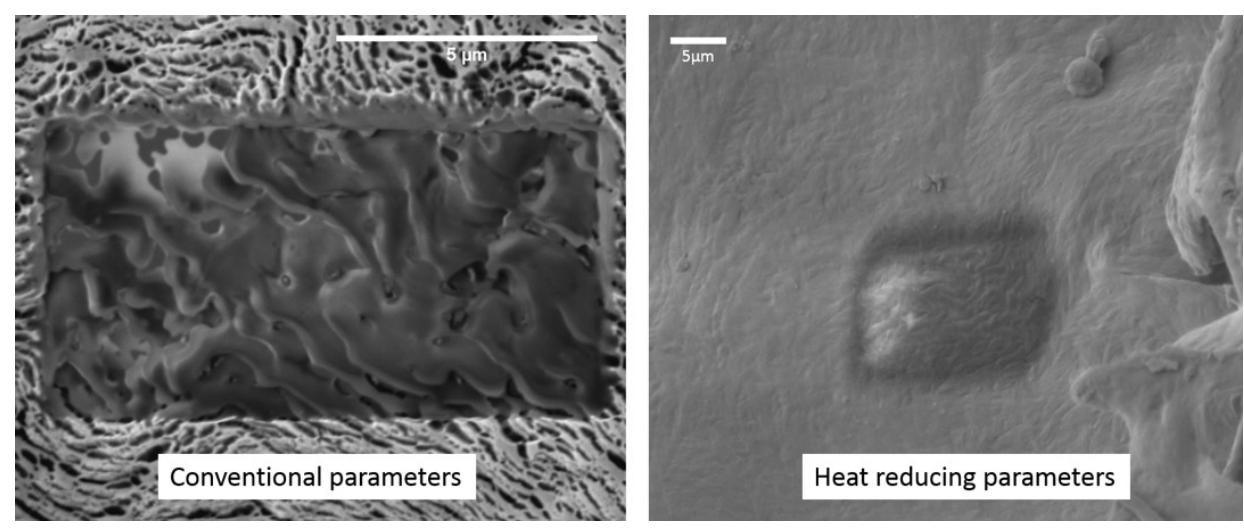

Figure 2. SEM images showing FIB prepared crosssections in collagen with conventional parameters (left) and heat reducing parameters (right). 